\title{
Type Ia Supernovae and Supersoft X-ray Sources
}

\author{
L. R. Yungelson \\ Institute of Astronomy, RAS, \\ 48 Pyatnitskaja Str., 119017 Moscow, Russia \\ email: lev.yungelson@gmail.com
}

\begin{abstract}
The rates of SNe Ia for double-degenerate and single-degenerate scenarios are computed for the models of spiral and elliptical galaxies. The number of nuclear burning white dwarfs (NBWDs) is traced. The data favors the double-degenerate scenario and suggests a lower number of NBWDs per unit mass in ellipticals. Their lower average mass is one of the reasons for the difference in the number of supersoft X-ray sources observed in the galaxies of different types.
\end{abstract}

Keywords. stars: binaries, supernovae $-\mathrm{X}$-rays: binaries

\section{Introduction}

While there exists an agreement that SNeIa result from thermonuclear explosions of white dwarfs (WD) which reach the Chandrasekhar mass $M_{C h} \approx 1.38 M_{\odot}$ (Hoyle \& Fowler 1960), the process of accumulation of $M_{C h}$ remains an enigma. It was suggested that WDs may accumulate $M_{C h}$ via accretion in semidetached binaries (singledegenerate scenario, SD, Schatzman 1963), merger of binary WDs with $M_{1}+M_{2} \gtrsim M_{C h}$ (double-degenerate scenario, DD, Webbink 1979), accretion of wind matter in symbiotic stars (Truran \& Cameron 1971). It is also hypothesized that accretion of He onto sub-Chandrasekhar CO WD may lead to SNe Ia via double-detonation (Livne 1990).

However, at the moment, only the SD- and DD-scenarios may be discussed as viable ones. Accretion in wide symbiotic systems is most probably not efficient enough for accumulation to $M_{C h}$. The double-detonation scenario, though claimed to provide the "correct" delay-time distribution of SNe Ia (DTD) and provide a significant fraction of the total SNe Ia rate (Ruiter et al. 2011), very strongly depends on the assumed efficiency of He-accumulation (Piersanti et al., these proceedings). Also, models do not fit observables, due to the presence of He-burning products in the outer layers of ejecta.

The count of supersoft X-ray sources (SSSs) in external galaxies may provide certain clues to the problem of progenitors of SNe Ia (Di Stefano 2010, Gilfanov \& Bogdán 2010). SSSs, which are deemed to be nuclear burning white dwarfs (NBWDs, van den Heuvel et al. 1992), may be direct precursors of SNeIa in the SD-scenario. In the DD-scenario, a binary may be an SSS when the first-formed WD accretes matter from the stellar wind of the precursor of the second WD. The number of observed SSSs provides a lower limit of the number of NBWDs, since the latter do not necessarily radiate in X-ray.

Below, we consider the relations between the rates of formation of NBWDs, SNe Ia, and SSSs in a model stellar system with a constant star formation rate $\dot{M}_{\star}=8 \mathrm{M}_{\odot} \mathrm{yr}^{-1}$ for $10 \mathrm{Gyr}$, and for a model in which the same mass of stars is formed in a 1-Gyr-long star-formation burst, i.e. for toy-models of a spiral and an elliptical galaxy, respectively. We test also the influence of CE parameters and the mass accumulation efficiency on the DTD. Our assumptions are described in Yungelson (2010). Note, we do not consider effects like "stripping" or "companion reinforced attrition process". 

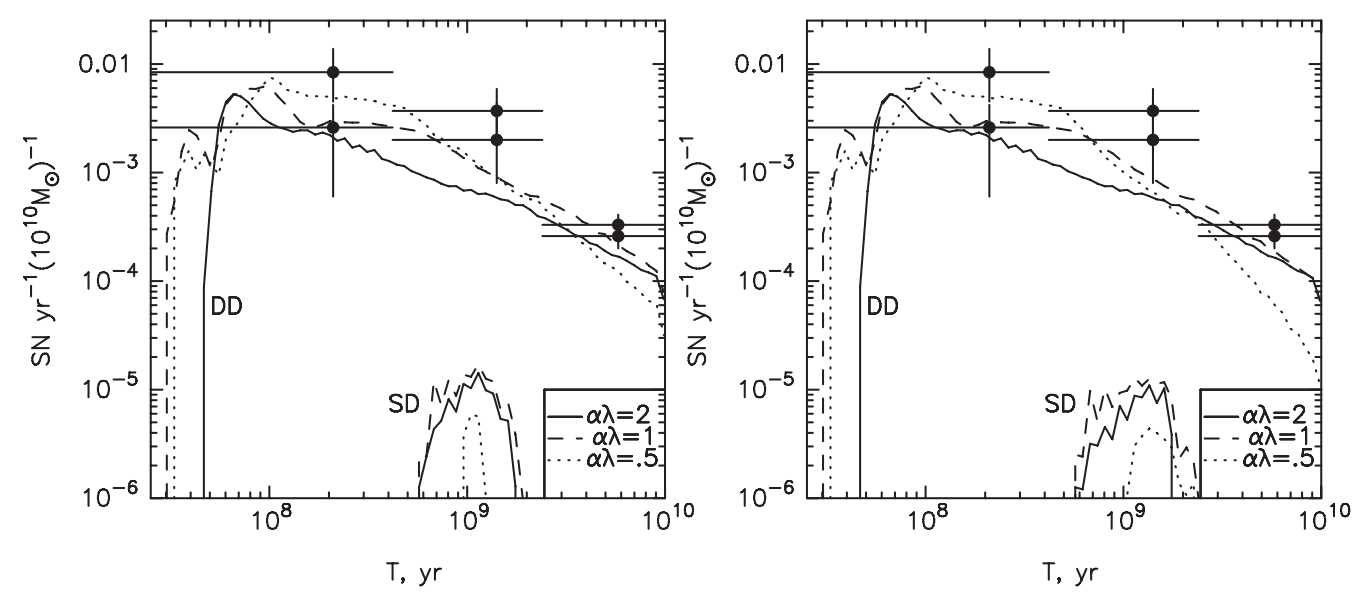

Figure 1. Model DTD for DD- and SD-scenario compared to observational data (Maoz et al. 2011). Two sets of the latter correspond to different models of the dust. Left panel $-\mathrm{H}$ - and He-accumulation efficiency after Prialnik \& Kovetz (1995) and Iben \& Tutukov (1996), right panel - efficiency after Hachisu et al. (1999) and Kato \& Hachisu (2004).

\section{Results}

In Figure 1 we compare the DTD for DD and SD-scenarios obtained for different values of the common envelope (CE) efficiency and donor envelope binding energy parameter product $\alpha_{c e} \times \lambda$ in Webbink's (1984) equation for CE. The total dominance of DD mergers is clear, irrespective of assumptions on $\alpha_{c e} \times \lambda$ and efficiency of matter accumulation. The main reason for this is a narrow range of combinations of masses of components in semidetached systems which allows steady accumulation of the matter. Bearing in mind all uncertainties involved in population synthesis and in derivation of DTD from observations, the slope of the model DD DTD $\left(t^{-0.8}\right)$ compares well with the slope of DTD curve $\left(t^{-1.2 \pm 0.3}\right.$ at $\left.t \gtrsim 400 \mathrm{Myr}\right)$ derived by Maoz et al. (2011) for SNeIa at $0<z<1.45$.

Figure 2 shows results of computations for two model stellar systems. The formation rate of close binary WDs reflects the SFR; hence, SNe Ia in the DD-scenario start at the age of several tens of Myr when the most massive pairs of WDs begin to form. In the "spiral" galaxy, binary WDs form continuously and the DD-SNe Ia rate permanently increases, since both "old" initially relatively wide pairs and "young" relatively close ones contribute to the merger rate. In the "elliptical" galaxy, the reservoir of binary WDs created in the star-formation burst gradually "melts", and soon after cessation of star formation, the DD-SNe Ia rate starts to decline.

In semidetached systems SNe Ia are delayed by $\sim 10^{9}$ yr respective to star formation. After a star-formation burst, semidetached systems in which a CO WD is able to accumulate to $M_{C h}$ form and exist only over a limited time span of several Gyr (Canal et al. 1996 and numerous later papers). In our model, SNe Ia occur in binaries with $M_{w d, 0} \gtrsim 0.85 M_{\odot}$ and $M_{s g, 0} \gtrsim 1.4 M_{\odot}$ at the beginning of Roche-lobe overflow. Figure 2 shows that the formation rate of semidetached systems with NBWDs able to reach $M_{C h}$ is only a minor fraction of the total formation rate of systems with NBWDs, even in the relatively early stages of evolution. Semidetached systems with NBWDs form continuously, but in the case of a burst-like star formation, currently observed SSSs are not precursors of SNe Ia. The epoch of SNe Ia in semidetached systems is short: in the model "spiral" galaxy at $t=10 \mathrm{Gyr}$, SNe Ia occur in stars formed approximately $600 \mathrm{Myr}$ to 2.5 Gyr ago. 

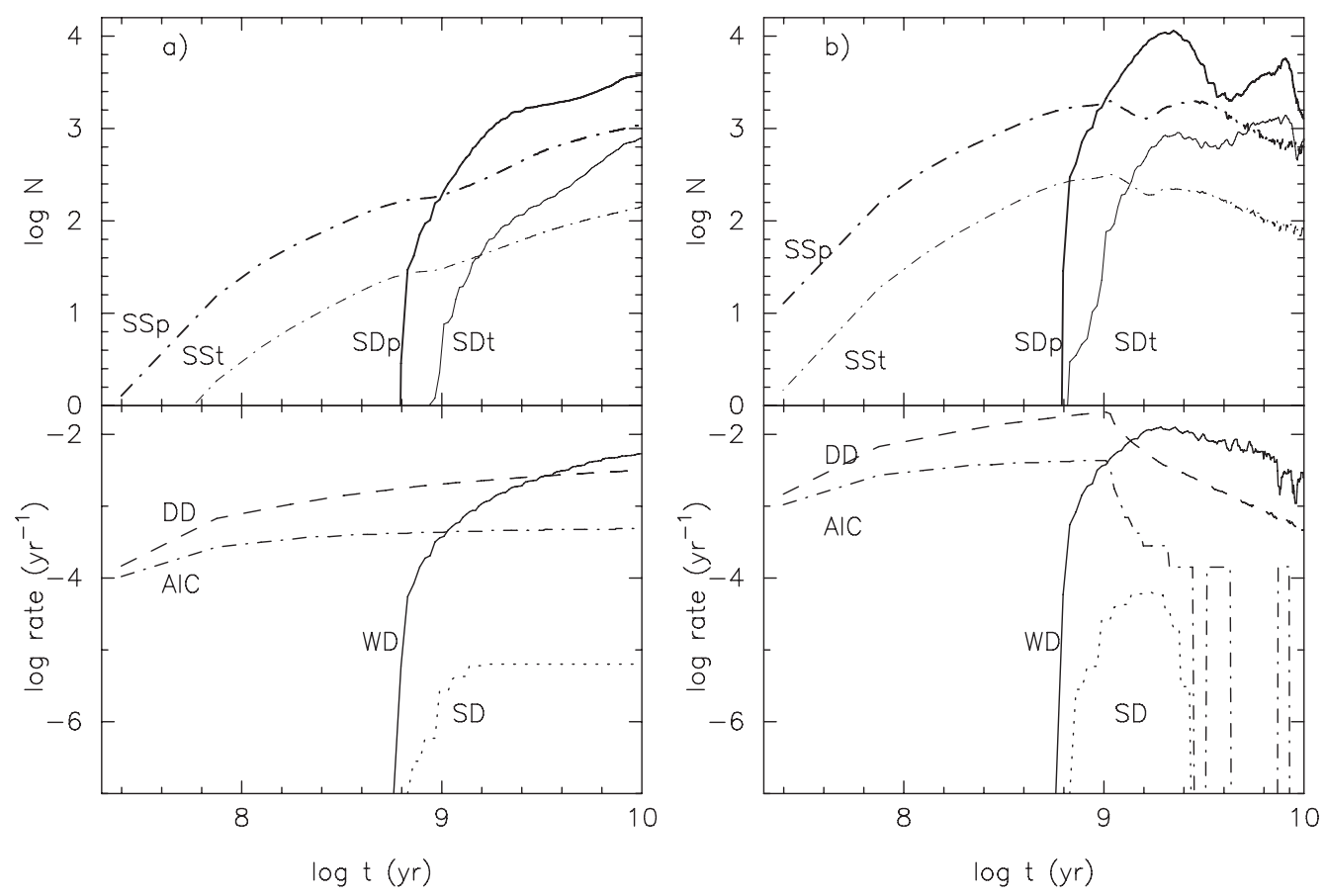

Figure 2. Panel (a), lower part - evolution of the rates of SNe Ia in DD-scenario (dashed line) and in SD-scenario (dotted line), formation of NBWD (solid line), AICs in symbiotic stars in the model of a "spiral" galaxy. Upper part - evolution of the numbers of permanent (SSp) and transient (SSt) SSS in symbiotic stars and permanent (SDp) and transient (SDt) sources in semidetached systems. Panel (b) shows evolution of the same rates and numbers in the model of an "elliptical" galaxy.

The phenomenon of symbiotic stars is associated predominantly with wide binaries, in which WDs form neither through RLOF nor CE. As the donor begins to ascend the giant branch, its stellar wind is weak and in a binary with $P_{\text {orb }} \sim(100-1000)$ day accretion is extremely inefficient. A newborn NBWD first becomes an unstable burner and, typically, it may become a stationary burner only shortly before the loss of the envelope by the companion (Yungelson et al. 1995). The amount of matter which may be retained by the WD is as a rule $\lesssim 0.1 M_{\odot}$ (Lü et al. 2006). An implication of this is that CO WDs with $M_{0} \lesssim 1.1-1.2 M_{\odot}$ hardly reach $M_{C h}$, while more massive ONeMg WD experience accretion-induced collapses (AIC, Nomoto \& Kondo 1991). AIC's may explain the weakest peculiar SNe Ia (Metzger et al. 2009). Evolution of the rate of AIC's in the models is also shown in Figure 2.

In Figure 3 we show the distribution of NBWDs over masses in two models. Distributions sum up both transient and permanent burners. At any time the latter dominate due to longer lifetimes. In the systems with subgiants the fraction of outbursting systems is initially low, since they first start to overfill their Roche lobes as relatively massive stars with high $\dot{M}$. Later, the fraction of systems in which the rate of accretion is lower than the limit for stable H-burning increases, since the systems with massive donors finish their evolution quickly. At $t=10 \mathrm{Gyr}$, the number of NBWDs in a "spiral" galaxy exceeds the number in an "elliptical" galaxy of the same mass, and masses of the WDs are also higher (Figure 3). Relatively well studied nearby spiral galaxies contain $\sim 100$ SSSs each, while elliptical galaxies host only $\sim 10$ SSSs per galaxy (Di Stefano 2010). Taking into account that Chandra is able to observe in these spiral galaxies only SSSs 


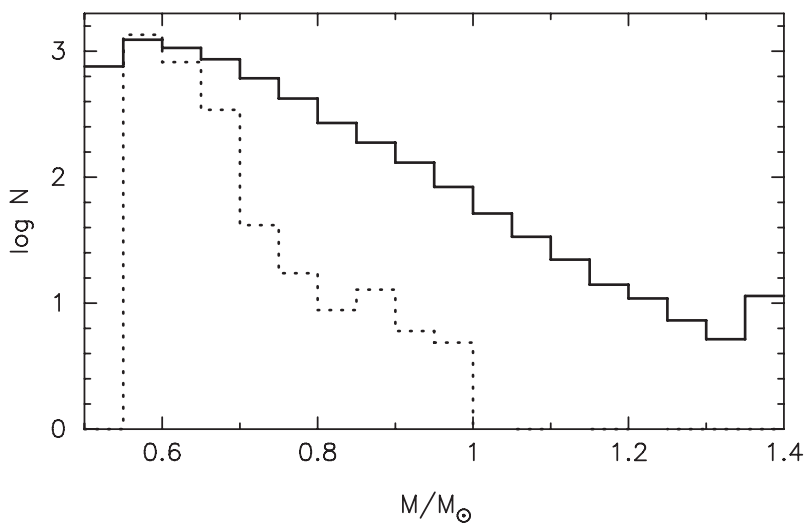

Figure 3. Mass spectrum of NBWDs in the model systems with continuous star formation (solid line) and with initial starburst (dotted line). The histograms show total contribution of semidetached and symbiotic systems.

with $M_{w d} \gtrsim(1.0-1.2) M_{\odot}$ and objects with $M_{w d} \gtrsim 0.8 M_{\odot}$ in elliptical galaxies (Di Stefano 2010), the number of NBWDs in the models at least qualitatively agrees with the one expected from observations. This brings to conclusion that the model in which the rate of SNe Ia is defined by the DD-mechanism and which is reasonably consistent with the observationally inferred DTD, is also able to explain, at least partially, the difference in the numbers of SSSs in spiral and elliptical galaxies.

The author acknowledges support from the IAU, RFBR (grant No. 10-02-00231), and the Program of the Russian Academy of Sciences "Origin and evolution of stars and galaxies".

\section{References}

Canal, R., Ruiz-Lapuente, P., \& Burkert, A. 1996, ApJ Lett., 456, L101

Di Stefano, R. 2010, ApJ, 712, 728

Gilfanov, M. \& Bogdán, Á. 2010, Nature, 463, 924

Hachisu, I., Kato, M., \& Nomoto, K. 1999, ApJ, 522, 487

Hoyle, F. \& Fowler, W. A. 1960, ApJ, 132, 565

Iben, I., Jr. \& Tutukov, A. V. 1996, ApJS, 105, 145

Kato, M. \& Hachisu, I. 2004, ApJ Lett., 613, L129

Livne, E. 1990, ApJ Lett., 354, L53

Lü, G., Yungelson, L., \& Han, Z. 2006, MNRAS, 372, 1389

Maoz, D., Mannucci, F., Li, W., Filippenko, A. V., Della Valle, M., \& Panagia, N. 2011, MNRAS, 412,1508

Metzger, B. D., Piro, A. L., \& Quataert, E. 2009, MNRAS, 396, 1659

Nomoto, K. \& Kondo, Y. 1991, ApJ Lett., 367, L19

Prialnik, D. \& Kovetz, A. 1995, ApJ, 445, 789

Ruiter, A. J., Belczynski, K., Sim, S. A., Hillebrandt, W., Fryer, C. L., Fink, M., \& Kromer, M. 2011, MNRAS, 1282

Schatzman, E. 1963, Star Evolution, 389

Truran, J. W. \& Cameron, A. G. W. 1971, Ap\&SS, 14, 179

van den Heuvel, E. P. J., Bhattacharya, D., Nomoto, K., \& Rappaport, S. A. 1992, Astron. Astrophys., 262, 97

Webbink, R. F. 1979, IAU Colloq. 53: White Dwarfs and Variable Degenerate Stars, 426

Webbink, R. F. 1984, ApJ, 277, 355

Yungelson, L., Livio, M., Tutukov, A., \& Kenyon, S. J. 1995, ApJ, 447, 656

Yungelson, L. R. 2010, Astron. Lett., 36, 780 